\title{
Cytohesin-2 Is Upregulated in Malignant Melanoma and Contributes to Tumor Growth
}

\author{
Yoon Jin Lee, Jin Ho Bae ${ }^{1}$, Woo Il $\mathrm{Kim}^{1}$, Jun Hyuk Kim², Sung Woo CHO, Sang Hoon Lee ${ }^{3}$, \\ Jin Seok Jeon, Hae Seon Nam, Seon Hwa Lee, Sang Han Lee, Moon Kyun Cho ${ }^{1}$ \\ Molecular Cancer Research, Soonchunhyang University College of Medicine, Cheonan, ${ }^{1}$ Department of Dermatology, Soonchunhyang \\ University Seoul Hospital, Seoul, ${ }^{2}$ Department of Plastic and Reconstructive Surgery, Soonchunhyang University Cheonan Hospital, \\ Cheonan, ${ }^{3}$ Department of Dermatology, Soonchunhyang University Bucheon Hospital, Bucheon, Korea
}

\section{Dear Editor:}

As human life expectancy is increased, cancer has been as a critical public health problem in today's world. In particular, the incidence of skin cancers has dramatically increased. Among the various types of skin cancers, malignant melanoma (MM), which is characterized by rapid proliferation and numerous metastasis, is the most aggressive type of skin cancer. MM accounts for a large proportion of deaths from skin cancers, because of its tendency to metastasize. Cancer cell migration is an important process in tumor cell invasion and metastasis.

Cytohesin-2 is a member of the cytohesin family that forms a subfamily of guanine nucleotide exchange factors (GEFs) for ADP-ribosylation factors (ARFs) that belong to the family of Ras GTPase superfamily ${ }^{1,2}$. As ARFs are involved in controlling several essential functions, such as cytoskeletal organization, cell migration, and signaling, cytohesins are key regulators of these processes ${ }^{2,3}$. There has recently been an increase in reports claiming that Cytohesin-2 plays important roles during physiological and pathological processes. Cytohesin-2 promotes HepG2 cell growth through the insulin-like growth factor pathway, and affects angiogenesis by regulating the internalization of vascular endothelial growth factor receptor-2 in endothelial cells and vessel permeability ${ }^{4}$. Cytohesin- 2 was expressed more in colorectal cancers and hepatocellular carcinoma (HCC) when compared to non-tu- morous tissues adjacent to carcinoma ${ }^{5,6}$. In addition, the up-regulation of Cytohesin-2 expression was found to be related with the proliferation, migration, and vascular invasion of tumor cells $\mathrm{s}^{5,7}$, and the prognosis of HCC patients ${ }^{6}$. However, there are no reports yet on the role of Cytohesin-2 in MM.

In accordance, we investigated the expression level of Cytohesin-2 in six cutaneous MM tissues. Samples were obtained from 4 nodular melanoma and 2 acral lentiginous melanoma. The Institutional Review Board of Cheonan Soonchunhyang University Hospital reviewed and approved this research protocol, which involved the use of tissue samples (IRB no. SCHCA 2013-07-006). To determine the expression levels of Cytohesin-2 proteins in $M M$ and normal skin tissues, Western blot analysis was performed. As shown in Fig. 1A, Cytohesin-2 was expressed in $\mathrm{MM}$, but rarely expressed in normal skin tissues. The relative protein expressions were statistically significant $(p<0.05)$ (Fig. 1B). Data showed a median (interquartile range) of $1.0726(0.94 \sim 1.19)$ and 0.7628 $(0.49 \sim 1.14)$ for $M M$ and normal skin tissues. We also evaluated expression of Cytohesin-2 in the tissues of six melanoma patients through immunohistochemical staining. Representative immunohistochemistry (IHC) staining results for Cytohesin-2 in normal skin and skin tumor tissues are shown in Fig. 1C and D. Significantly stronger staining intensities of Cytohesin-2 in MM tissues were found com-

\footnotetext{
Received January 5, 2018, Revised March 2, 2018, Accepted for publication March 7, 2018
}

Corresponding author: Moon Kyun Cho, Department of Dermatology, Soonchunhyang University Seoul Hospital, 59 Daesagwan-ro, Yongsan-gu, Seoul 04401, Korea. Tel: 82-2-709-9368, Fax: 82-2-709-9374, E-mail: mkcho@schmc.ac.kr ORCID: https://orcid.org/0000-0002-2180-9365

This is an Open Access article distributed under the terms of the Creative Commons Attribution Non-Commercial License (http://creativecommons.org/ licenses/by-nc/4.0) which permits unrestricted non-commercial use, distribution, and reproduction in any medium, provided the original work is properly cited.

Copyright (C) The Korean Dermatological Association and The Korean Society for Investigative Dermatology 
A
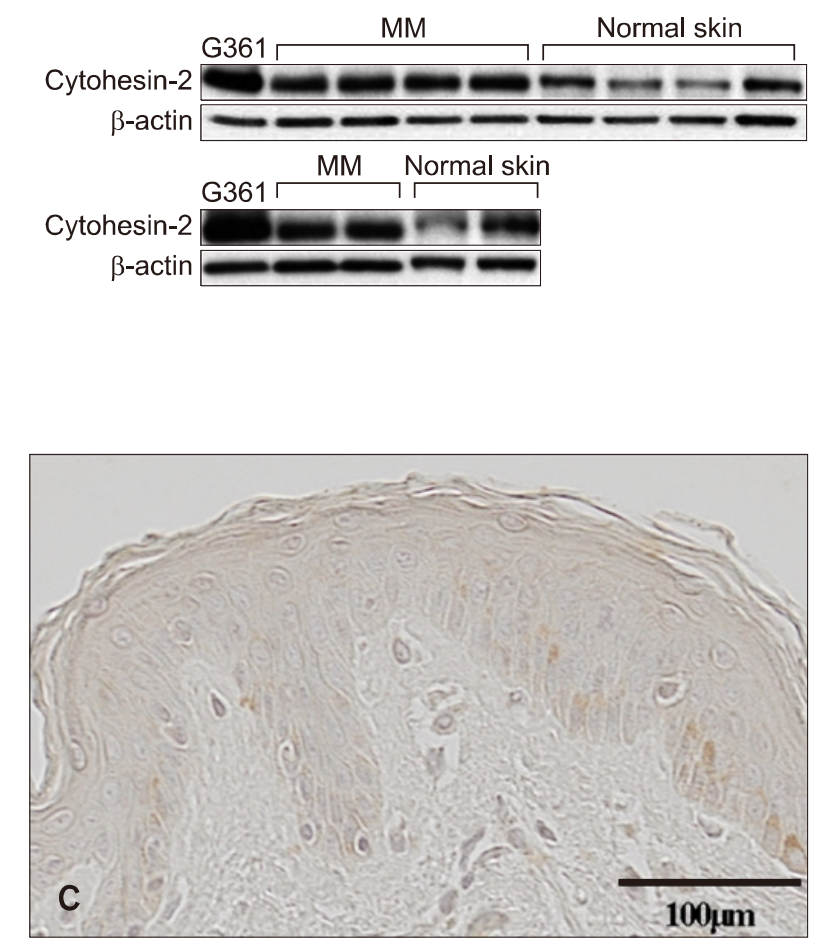
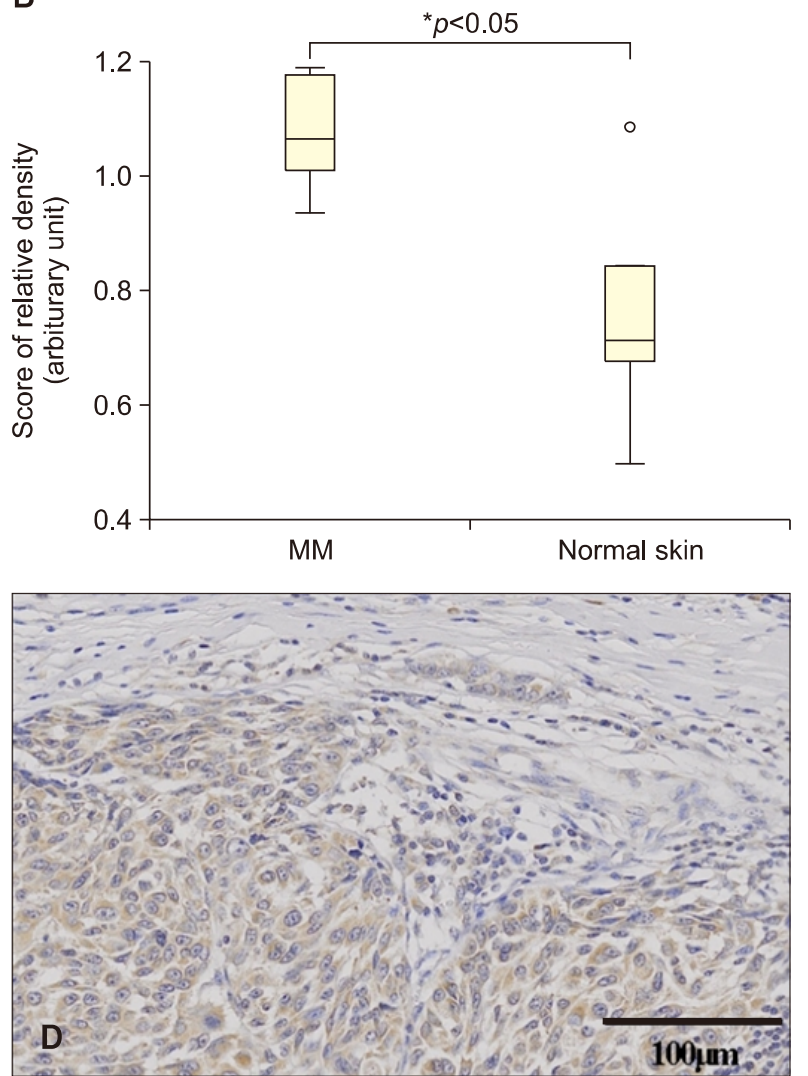

Fig. 1. Western blot analysis of Cytohesin-2 in malignant melanoma. (A) Cytohesin-2 was expressed in malignant melanoma (MM) tissues, but was decreased in normal human skin tissues. (B) The relative protein expression of Cytohesin-2 according to the Mann-Whitney U-test was analyzed. Representative immunohistochemistry (IHC) staining for Cytohesin-2 protein expression in paraffin-embedded normal skin tissue and MM. Brown color spots were positively stained cells. (C) Negative staining of Cytohesin-2 in normal skin (IHC stain, $\times 400)$. (D) Mild to moderate staining of Cytohesin-2 in MM (IHC stain, $\times 400)$. $\beta$-actin, beta-actin.

pared to those in normal skin tissues.

To find downstream molecules of the Cytohesin-2 signaling in G361 cells, the levels of p-ERK and p-AKT were assessed by Western blotting following transfection with Cytohesin-2-specfic siRNA. As shown in Fig. 2A, downregulation of Cytohesin-2 expression was observed at 48 hours after siRNA treatment. Treatment of G361 cells with siRNA inhibited the phosphorylation of extracellular signal-regulated protein kinases 1 and 2 (ERK1/2) and AKT. These results indicate that Cytohesin-2 may stimulate MEK/ERK1/2 and/or phosphatidylinositol-3-kinase (PI3K)/AKT pathways in G361 cells. Increased rate of apoptosis was found in G361 cells after transfection with Cytohesin2-specific siRNA. Based on MTT assay, cell viability was decreased in a time-dependent manner following knockdown of Cytohesin-2 (Fig. 2B). In the flow cytometric analysis, cells transfected with Cytohesin-2-specific siRNA tended to decrease the proportion of cells in the G1 and S phases compared to control cells, while that in the G2/M phase tended to increase (Fig. 2C). In apoptosis assay, three different cell populations were detected after Annexin V-PE staining, including live cell group (lower left panel), early apoptotic cell group (lower right panel), and late apoptotic cell group (top right panel). An increase in apoptotic cell population was observed following transfection with siRNA (Fig. 2D). These results indicated that Cytohesin-2 may promote the survival of G361 cells. We examined the silencing effect of Cytohesin-2 on cell migration using a scratch wound healing assay in G361 cells. As shown in Fig. 2E, silencing of Cytohesin-2 significantly decreased the wound filling ability of G361 cells. Cells treated with Cytohesin-2-specific siRNA had much slower wound filling ability than untreated controls. As shown in Fig. 2F, knockdown of Cytohesin-2 significantly exhibited much less colony formation than untreated controls. This result indicated that Cytohesin-2 may be involved in the tumor cell aggressiveness such as cancer cell migration and tumorigenic potentials. 
A

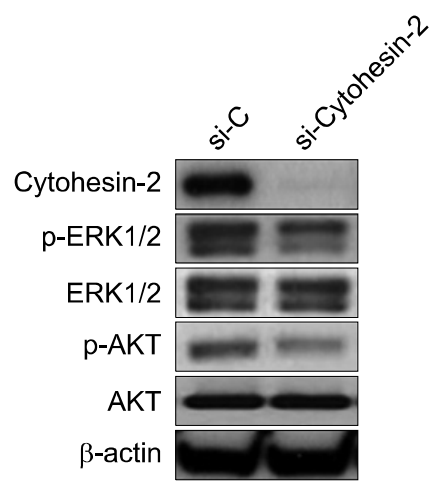

C

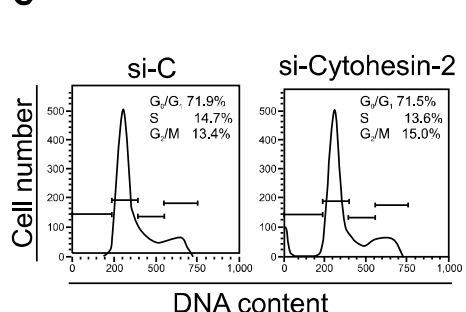

DNA content

E

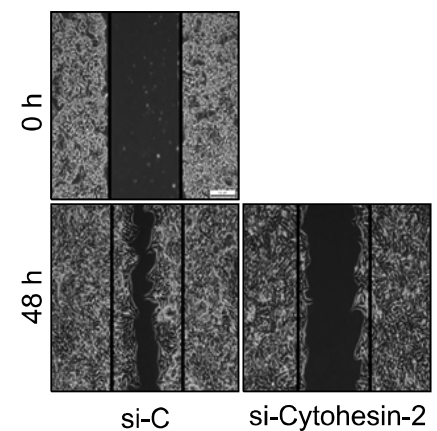

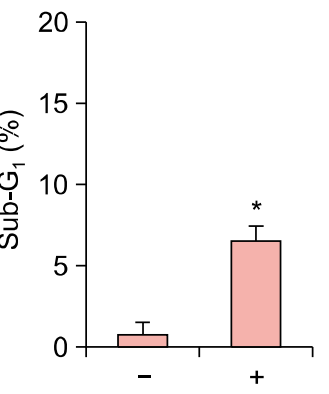

si-Cytohesin-2

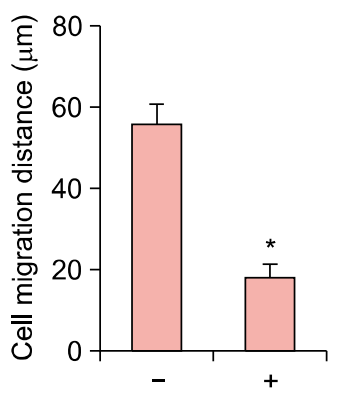

si-Cytohesin-2
B

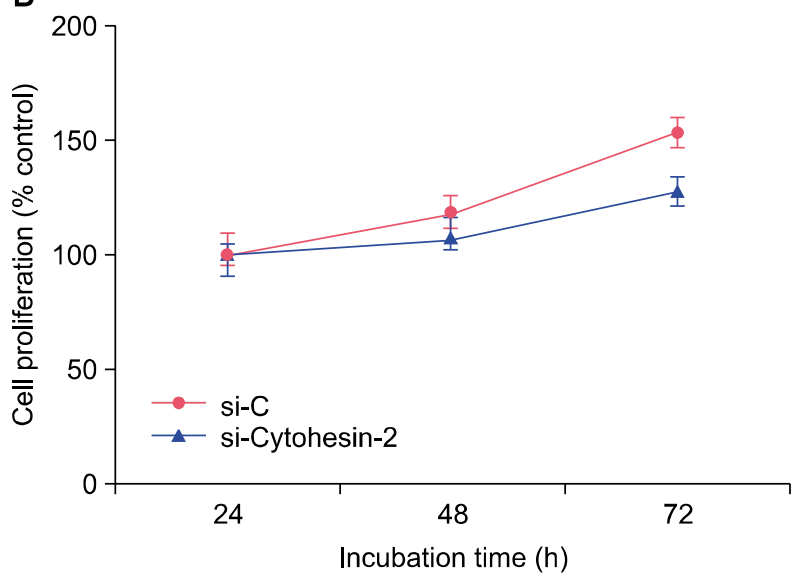

D
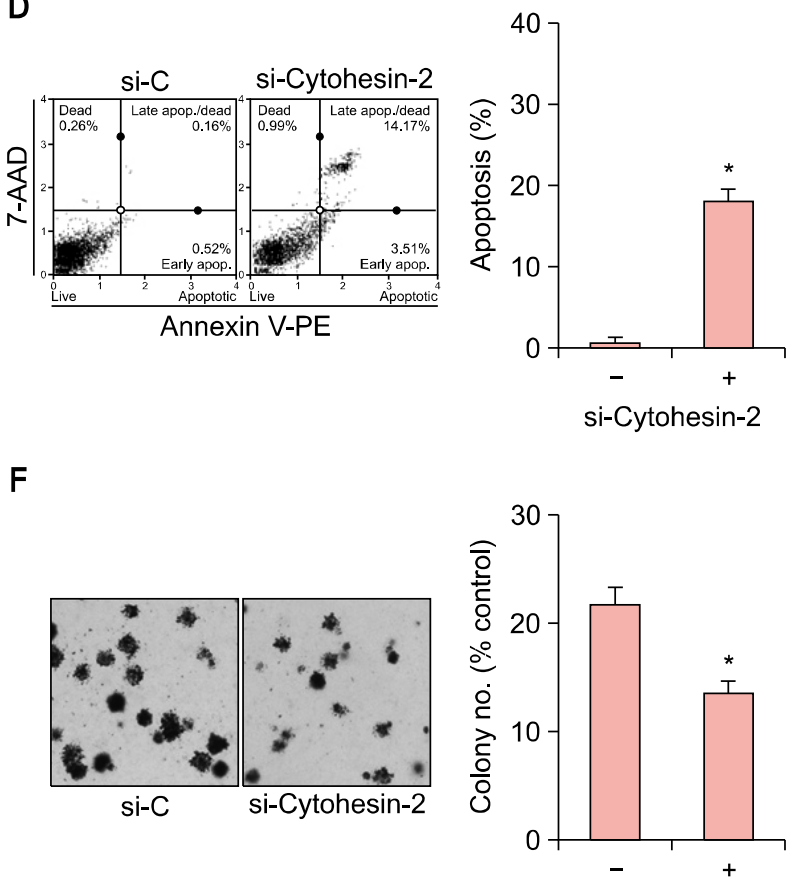

si-Cytohesin-2

Fig. 2. G361 cells were transfected with Cytohesin-2-specific siRNA. (A) Western blot of Cytohesin-2, p-ERK1/2, ERK, p-AKT and AKT protein. (B) The percentage of viable cells was measured by MTT assay. (C) Cell distribution in G0/G1, S, and G2/M phases was analyzed using flow cytometry after staining with propidium iodide $(20 \mu \mathrm{g} / \mathrm{ml})$. (D) The percentage of apoptotic cells after Annexin V-PE binding was analyzed using a Muse Cell Analyzer. (E) Representative images of the wound healing assay in G361 cells. (F) Colony formation assay for G361 cells transfected with control and si-Cytohesin-2. Error bars represent mean \pm standard deviation for three independent experiments. ${ }^{*} \mathrm{p}<0.05$ compared to respective controls. $\beta$-actin: beta-actin, si-C: StealthTM RNAi control, si-Cytohesin-2: small-interfering Cytohesin-2, p-: phospho-, ERK: signal-regulated protein kinases, 7-AAD: 7-aminoactinomycin D.

The oncogenic roles of Cytohesin-2 have been observed in hepatocellular carcinomas and colorectal cancers, but the expression and signaling effects of Cytohesin-2 expression has not yet been studied in skin cancers. In this study, we have identified high expression of Cytohesin-2 in $\mathrm{MM}$, using $\mathrm{IHC}$ staining and Western blotting. Considering a well-established role of Cytohesin-2 as an endogenous GEF for ARFs, this finding prompted us to further inves- tigate the importance of Cytohesin-2 expression in cutaneous MM. Consistent with previous studies, we observed the up-regulation of Cytohesin-2 expression in MM. The finding that Cytohesin-2 is involved in cell migration and invasion has been reported in several cancer studies ${ }^{5,7}$. Up-regulation of Cytohesin-2 in Madin-Darby canine kidney cells has been associated with rapid flattening of cells at the periphery and the formation of large invadopodia, 
which can also enhance cell motility and cause individual cells to dissociate from their neighbors ${ }^{7}$. Pan et al. ${ }^{5}$ have reported that migration and invasion of colorectal cancer cells are inhibited when Cytohesin-2 is inhibited by SecinH3 (Sec7 domain-binding selective antagonist).

In addition, several reports have suggested that Cytohesin2 can regulate cell proliferation and motility through ERK $1 / 2^{8,9}$ and $\mathrm{PI} 3 \mathrm{~K}$ signaling pathway in vitro ${ }^{10}$. Accumulating evidence indicates that Cytohesin- 2 can cross-communicate in cell signaling events through MEK/ERK $1 / 2$ and/or PI3K/AKT pathways, which are necessary for cellular responses such as cell survival, proliferation, and migration. Cytohesin-2 is involved in the activation of AKT pathway and endothelial cell proliferation ${ }^{10}$. Furthermore, it has been reported that Cytohesin-2 promotes PI3K-dependent ARF6 activation, and then leads to phosphorylation of ERK $1 / 2$ in preadipocyte migration ${ }^{9}$.

Considering the oncogenic roles of Cytohesin-2 in MM, the understanding of its function might potentially contribute to the development of novel pharmaceutical targets for enhancing the therapeutic efficacy of skin cancers.

\section{ACKNOWLEDGMENT}

This work was supported by the Soonchunhyang University Research Fund.

\section{CONFLICT OF INTEREST}

The authors have nothing to disclose.

\section{ORCID}

Yoon Jin Lee, https://orcid.org/0000-0002-0911-2485

Jin Ho Bae, https://orcid.org/0000-0001-8075-6438

Woo II Kim, https://orcid.org/0000-0001-7127-2992

Jun Hyuk Kim, https://orcid.org/0000-0002-0266-8480

Sung Woo CHO, https://orcid.org/0000-0002-7206-798X
Sang Hoon Lee, https://orcid.org/0000-0002-7146-3702 Jin Seok Jeon, https://orcid.org/0000-0003-2421-2289 Hae Seon Nam, https://orcid.org/0000-0002-2498-2147 Seon Hwa Lee, https://orcid.org/0000-0002-7516-2122 Sang Han Lee, https://orcid.org/0000-0001-6407-9959 Moon Kyun Cho, https://orcid.org/0000-0002-2180-9365

\section{REFERENCES}

1. Moss J, Vaughan M. Molecules in the ARF orbit. J Biol Chem 1998;273:21431-21434.

2. Kolanus W. Guanine nucleotide exchange factors of the cytohesin family and their roles in signal transduction. Immunol Rev 2007;218:102-113.

3. Casanova JE. Regulation of Arf activation: the Sec7 family of guanine nucleotide exchange factors. Traffic 2007;8:14761485.

4. Lim J, Zhou M, Veenstra TD, Morrison DK. The CNK1 scaffold binds cytohesins and promotes insulin pathway signaling. Genes Dev 2010;24:1496-1506.

5. Pan T, Sun J, Hu J, Hu Y, Zhou J, Chen Z, et al. Cytohesins/ ARNO: the function in colorectal cancer cells. PLoS One 2014;9:e90997.

6. Xu K, Gao J, Yang X, Yao Y, Liu Q. Cytohesin-2 as a novel prognostic marker for hepatocellular carcinoma. Oncol Rep 2013;29:2211-2218.

7. Santy LC, Casanova JE. Activation of ARF6 by ARNO stimulates epithelial cell migration through downstream activation of both Rac1 and phospholipase D. J Cell Biol 2001;154:599-610.

8. Muralidharan-Chari V, Hoover H, Clancy J, Schweitzer J, Suckow MA, Schroeder V, et al. ADP-ribosylation factor 6 regulates tumorigenic and invasive properties in vivo. Cancer Res 2009;69:2201-2209.

9. Davies JC, Tamaddon-Jahromi S, Jannoo R, Kanamarlapudi $\mathrm{V}$. Cytohesin 2/ARF6 regulates preadipocyte migration through the activation of ERK1/2. Biochem Pharmacol 2014;92:651660.

10. Mannell HK, Pircher J, Chaudhry DI, Alig SK, Koch EG, Mettler R, et al. ARNO regulates VEGF-dependent tissue responses by stabilizing endothelial VEGFR-2 surface expression. Cardiovasc Res 2012;93:111-119. 